\title{
Cognitive Linguistics Translation View and its Enlightenment on Translation Abilities
}

\author{
Linlin Sun \\ Department of English, Liaoning College of Science and Engineering, Jinzhou, 121000, China
}

Keyword: Cognitive Linguistics, Translation Theory, Translation Abilities enlightenment.

\begin{abstract}
The main core of translation is to develop students' translation ability, from the view of cognitive linguistics, there is a close relationship between cognitive activity and translation capability; cognitive linguistics translation theory provides basis for the translation teaching and makes translation a new perspective. Takes cognitive linguistics-based translation teaching mode to cultivate students' translation ability, to a certain extent, can improve some of the problems generated by the current translation teaching, this paper mainly analyze the enlightenments of cognitive linguistics translation theory and its translation abilities cultivation.
\end{abstract}

\section{Cognitive Linguistics Translation Theory}

Cognitive linguistics and cognitive psychology, linguistics combining a marginal discipline, for the current translation teaching, the cognitive linguistics played a significant role in the culture of teaching and translation competence are significant role in promoting. Cognitive linguistics is that the translation can relate to many levels, it is of its time, it can affect their state of development, the translation itself is not an independent existence, translation behavior in the real state makes the Cognition Linguistics affected to some degree. Cognitive Linguistics has a body of knowledge, it receives information from the various interactions coincide, which also makes the translation will be affected, in addition, an important factor is the translation of the reader itself, the presence of translation an important reason is to let the reader understand some of the knowledge from many readers as an interpreter indispensable subject in the translation of knowledge and learned construction will involve a variety of behaviors, leading to translate the behavior itself is also affected.

Views on translation in cognitive linguistics authors, works and readers have higher requirements, it is focusing on interaction between the three, through the translator to understand the work itself generates its own cognitive system, which is the concept of translation He stressed out by the experience of the translator subjectivity performance in the real world. Translation Concept Although not explicitly stated in relation to the author essays configuration works with readers, but which referred the authors could not with their own subjective feelings to assess the work, but to consider the work itself and in conjunction readers to ponder on the work, the translation interactive reflected, in which the reader's influence on translation would have a negligible effect. In short, cognitive linguistics translation theory not only recognizes, describes the cognitive activities in the translation of this behavior in the objective existence of translators also made certain requirements. Cognitive cognitive linguistics translation activities can promote the related translation researchers and teachers discover some new problems in translation teaching, and the use of cognitive linguistics issue will be resolved well, it is possible to improve the quality of teaching and promoting translation cognitive development of linguistics. 


\section{Malpractice of Translation Abilities Cultivation in Current Translation Teaching}

\section{Translation teaching lacks of systematic organization and theoretical guidance}

Translation Studies itself with high limitations, resulting in a lack of translation teaching system and organization theory. Translation Studies as a subject has been under linguistics, rather than as a separate subject exist, which makes the translation in the teaching process can not be used as a separate entity, but mixed with other linguistic subjects, greatly reduced translation teaching quality and timeliness. From our contact with translation teaching began to recognize things only language conversion surface, most people think is a kind of translation into another language or languages of procedure, while ignoring the cultural context of translation, and the translator language translation purposes, these factors have a great influence on the effect of the translation produced. They lack of knowledge translation activities, so that the language becomes a single and narrow, this lack of language translation of the senses lead us in the process of translation no specific knowledge, it lacks a certain amount of cultural knowledge accumulated, ignoring translation complexity and purpose, so that the translation process too mechanical and simplistic.

\section{Teachers are lack of professional translation knowledge and quality}

Translation Teaching high demand for teachers, it requires teachers to have professional translation teaching quality and teaching experience, but the current level of professionalism of the translation industry is not high, most teachers are not engaged in translation work, understanding of language translation nor specific in the teaching process to just a single language will be converted, without explanation and teaching theoretical knowledge. College Translation Teachers not have long-term experience as a translator, and even some teachers only held by graduates of linguistics, which allows teachers to a large extent the lack of proper understanding of the translation in the teaching process which led to students being misled teachers when the students would be too dependent on translation teaching translation and translation for their own lack of understanding of itself, and needs assessments will be confined to the pros and cons and make the translation into a language teaching assisted learning courses. Translation course itself is relatively complex, students generally difficult to know the specific meaning of the original from access to information, largely literal translation would make the articles and sentences to express the meaning is distorted, so teachers teaching students to translate He said translation is indispensable to understand the nature of the way, but with a lot of teachers in the teaching of translation experience individualism mark with his past to learn the way students learn, and learning a lot of students will not match the personality, leading to students' lack of interest in translation courses in the learning process, and unwilling to accept the teacher's teaching.

\section{Lack of specific awareness for translation}

In this stage of the translation teaching, many teachers and students to recognize specific translation is not in place, in the traditional view, the teacher is teaching that the purpose of the translation is to allow students to complete basic education, which in translation teaching for students when teachers often scripted, the contents of the textbook to teach students. Some basic knowledge of grammar, mostly by students themselves through self-learning materials and extracurricular hearing is completed, the teacher in the classroom to teach students only a few simple words and sentences, and then let the students to integrate knowledge learned translate a simple experience. In high school learning process, under the pressure of studies, most students will learn the knowledge translation as a means of studies that will be written foreign language translation of basic knowledge learned can have a good result. In the process of language learning university, students will learn is that the main basis of language translation can better survive in society, because in college graduate looking for work, many units of recruitment have some foreign language proficiency requirement, this allows students eager to have a foundation of language translation of understanding, rather than use their energies on the exact understanding of the translation. Each learning stage for arranging translation course very limited quantity, limited class so that students do not have enough learning opportunities, 
and lack of extensive and a lot of practice, leading students to translate the lack of specific knowledge.

\section{Students are in a passive position in the classroom}

While the existing teaching methods are under the formation of the new curriculum reform, but linguistics translation teaching and other teaching models are very different, not in a short time teaching models and learning methods change, so the translation teaching to a large extent the continuation of traditional teaching methods, that is to say, the students in the classroom is not have too much initiative. The traditional teaching model, students in the classroom for teachers is consistently talking about the contents of the hard memory, and rarely have the opportunity to express their views, not to mention the initiative in the classroom. But the essence of language translation, is the need to communicate student understanding of language in the process of translation, the language needs to be holistic and systematic organization of finishing students in the classroom so that students in a passive position no language initiative, resulting in a lack of interest in language translation, language translation can not be performed better learning.

\section{The Enlightenment of Cognitive Linguistics Translation Theory on Translation Abilities}

\section{Linguistics translation theory can be developed into a teaching mode}

Cognitive Linguistics Translation Theory put forward a macro translation process, contact between the language may be the correspondence between man and the text, this concept can be put in Translation Teaching in the formation of a new teaching mode. Translation Teaching in the content comprises at least two elements, one is for students to learn basic translation from the original understanding of the teacher's help edit the translation to be independent, and the other teachers in carrying out translation activities for students language conversion teaching, these two translations translation can help students develop interpersonal relationships. Existing language translation teaching there are some flaws, the purpose of translation is to enable students to experience a different culture in the learning process and passes Mandarin culture to the world, which is the language translation in a cultural transfer, linguistics translation development concept as a teaching mode can correct the existing problems in the teaching mode, and effective translation teaching for their vulnerability. Cognitive Linguistics Translation Theory to determine the cultivation of translation competence is central to the teaching of translation, on the basis of cognitive linguistics on translation teaching mode can be improved to a large extent so that the development of a new translation teaching teaching mode, teachers and students to combine comprehensive consideration to improve student learning in improving teaching quality of the premise down.

\section{Cognitive Linguistics Translation Theory takes the relationship with the real world experience as a philosophical basis}

Translator language translation is a very important concept, he is the process of translating the most important and most active body of experience and knowledge, we can say the translator cognitive linguistics and pushed to the dominance of the core translation activities. Translators need to have not only a language translation capabilities, but also the need for people to understand a language conversion capability and processing power of language in the translation process, will encounter many problems, these need to be translators coordination is good and processed in the best way, so as to the practical problems and theoretical knowledge combined with the translation work done better. Translation of "Two Worlds" and "harmony," "multiple interaction" and is considered to be an important part of cognitive linguistics, translation theory, the "Two Worlds" also includes the aforementioned translators need to have coordination , from the literal meaning, it also refers to two different concepts and the nature of the translation and the original, it is the basis of the relationship between real-world experience and expression, the translator should be noted that, for interactive multi-linguistic translation is very important, as a translator at the time of the individual articles and 
materials for translation is very boring, but the translation to multiple interactive exhibits to attract readers translations profound analysis and interpretation.

\section{Cognitive linguistics translation concept has some significance on classroom teaching}

In translation teaching mode of cognitive linguistics, the traditional teaching model will be broken, students in the classroom can occupy a dominant position, and extends from the teacher's teaching materials based on the extra-curricular, so that classroom teaching can have more basis genre. Traditional teacher reviews rigid translation students remember the way in which the teaching pattern is bound to be negative, is no longer the focus of attention of teachers to improve student achievement but in the ability to translate practical training high school students whether there is a substantial increase, many in literal translation, free-based materials will be eliminated, because the training is not teaching language skills to convert from actually improve students' translation ability. Focus on language translation "Translation of Discourse" is student-centered, will integrate such knowledge to write textbooks can allow students to refer to some translations translation assessment, and can add their own emotional, which is classroom teaching meaningful.

\section{Conclusion}

Cognitive Linguistics Translation Theory has been basically established in our country, but in the traditional translation teaching, teachers' teaching mode has deep imprint, so that teaching mode in a short time is difficult to be expanded and extended, resulting in translation or teaching there are many problems. But cognitive linguistics and language learning to provide students with the theoretical guidance, which was applied to translate into teaching so that students can have a more specific language translation of knowledge through teaching linguistics translation theory, the theory of teachers can be with practice, the students translation activities more effective guidance to improve students' translation ability.

\section{References}

[1] Wu Bo. Cognitive Linguistics Translation Theory and Its Implications for Translation Abilities. Journal of Sichuan foreign language institute, 2008(01) .

[2] Huang Yuan. Cognitive Linguistics Translation and its Enlightenment on Translation Abilities. Overseas English, 2011(07).

[3] Wang Lisi. Cognitive Linguistics and Translation Theory and Its Implications for Translation Teaching in Universities. Crazy English (Teachers' edition), 2015(02).

[4] Zhu Lifeng. Cognitive Linguistics and Translation Theory of Translation Abilities. Modern Science, 2009(12).

[5] Zhang Ting. Cognitive linguistics and translation theory and translation of Ability. Journal of Longdong College, 2010(09).

[6] Wu Xiaojun. On Construction of Interactive Teaching Model English translation. Foreign Languages Research, 2007(04). 